\title{
Lecciones de una experiencia de enseñanza de la Geografía asistida con ordenador
}

\author{
ANTONIO MORENO JIMÉNEZ *
}

\section{INTRODUCCIÓN}

La difusión de la informática constituye en la actualidad un ejemplo singular de socialización masiva de una tecnología muy poderosa y útil en innumerables facetas de la actividad humana. La enseñanza constituye un vehículo privilegiado en ese proceso porque, a través de ella, se puede dar a conocer esa innovación, incorporándola como un elemento habitual del medio cultural en que el joven se va a desenvolver. Al mismo tiempo la propia labor docente soporta el impacto de ella y ha de adaptarse para aprovechar las posibilidades que le ofrece.

En el campo concreto de la Geografía existe ya un cierto número de experiencias, e incluso valoraciones, del papel que la informática puede jugar en el proceso de aprendizaje (Shepherd, Cooper y Walker, 1980, Kent, 1983, Moreno et al., 1983, Reeve, 1984, Shepherd, 1985, Midgley y Walker, 1985) de las que se desprende que, junto a aspectos positivos evidentes, emergen igualmente problemas, pero que en todo caso parece ineludible la adopción en un futuro próximo de ese medio como un auxiliar más, al igual que las diapositivas, los mapas, etc.

\footnotetext{
* Universidad Autónoma de Madrid.
} 
Como recurso didáctico el ordenador puede ser empleado en una gama variada de formas; desde aquéllas en las que actúa como una ayuda en las exposiciones de clase (pizarra electrónica), hasta aquellas otras en las que realiza tareas bajo las órdenes directas de los alumnos, sin mencionar las relacionadas con temas de gestión docente (listas de alumnos, calificaciones, bibliotecas, etc.). El interés del autor por esta tecnología, surgido inicialmente con un horizonte exclusivamente investigador (cf. Vidal, Bosque y Moreno, 1983) se extendió al campo docente con una clara motivación: enriquecer el bagaje instrumental del que el geógrafo echa mano para enseñar su disciplina. El horizonte actual, en el que se divisa una creciente instalación de aulas informáticas con fines didácticos, hace particularmente recomendable difundir experiencias de enseñanza con ordenador que ilustren los modos o estrategias potencialmente asumibles por el profesor de Geografía (Cf. Moreno, 1988).

\section{EL CONTEXTO DE LA APLICACIÓN: EL TRABAJO DE INVESTIGACIÓN DE NIVEL ELEMENTAL}

La modalidad de empleo del ordenador que se adoptó fue la realización de simulaciones directamente por los alumnos. Más en concreto se trató de la ejecución de proyecciones demográficas en el contexto de un trabajo de curso, con lo que desde el punto de vista pedagógico se perseguía la confluencia o adición de los aspectos positivos de la enseñanza apoyada en proyectos de investigación geográfica (cf. Silk y Bowlby, 1981, y Beaumont y Williams, 1983) y en la informática. La experiencia fue realizada dentro de la asignatura de Geografía Humana que se imparte en el segundo curso del primer ciclo de licenciatura.

La orientación general del trabajo de curso obedecía a dos premisas: por un lado se trataba de iniciar al estudio de la geografía local (varios municipios dentro de una hoja del MTN $1 / 50.000$ de libre elección), siguiendo una tradición ya larga en nuestra disciplina, mediante una indagación continuada a lo largo del año académico y en sincronía con las explicaciones teóricas (examen del medio, la población, actividades económicas, etc.). Por otro se deseaba la aplicación integrada de las nociones, conceptos, representaciones gráficas y cartográficas introducidas en clase y el manejo de fuentes estadísticas publicadas. 
Dentro de ese contexto los objetivos asignados al ejercicio de proyección consistian en la comprensión del cambio poblacional (factores, componentes, dinámica, etc.), enfrentando a los alumnos con el problema de realizar un pronóstico de la evolución demográfica futura para todos y cada uno de los municipios de la zona elegida.

Esa tarea se insertaba dentro de una trama de actividades desarrolladas de forma coordinada por profesor y alumnos sobre el tema del crecimiento de la población. Tras la exposición de las fuentes, índices de crecimiento, evolución histórica de la población y las tasas, teoria de la transición demográfica, etc., los alumnos (en equipos de dos) debieron realizar un análisis de esos puntos para la zona respectiva.

Tras esa fase se suscitó la ejecución de las proyecciones. Las actividades programadas al respecto se pueden resumir así:

a) Exposición teórica general sobre las proyecciones de población; a ello se añadió un estudio más detallado del modelo de proyección a usar (fórmulas matemáticas, exigencias de información, especificación de las hipótesis de crecimiento, etc.), asi como un análisis en clase de publicaciones donde se había aplicado (con el propósito de ejemplificar sobre todo el discurso conducente al establecimiento de hipótesis).

b) Esta información de carácter conceptual y metodológico se completó con un pequeño cuaderno de datos cercanos al problema a resolver, incluyendo estadísticas, tasas y mapas sobre crecimiento, migraciones, etc., de la población española durante las últimas décadas, con objeto de iluminar el marco geográfico amplio en el que se habrían de situar las previsiones y tendencias.

c) Una breve introducción sobre el ordenador y sus prestaciones en Geografía.

d) Una demostración práctica del uso del programa. Para ello se empleó el aula informática del Centro de Cálculo de la UAM que disponía de un terminal del ordenador y pantallas grandes de visualización.

e) Realización personal de la proyección.

\section{EL PROGRAMA Y SU PUESTA A PUNTO EN UN ENTORNO INTERACTIVO}

Una consideración ineludible para la preparación del "software" didáctico consistía en los nulos conocimientos informáticos de los alumnos; 
más aún, tampoco era un objetivo el enseñar informática, sino más bien utilizarla al servicio de la enseñanza de la Geografía. Por otro lado, se asumía igualmente la necesidad de un manejo directo de los terminales del ordenador por los estudiantes, tanto para la resolución de su problema particular, como para desmitificar la máquina y aprovechar el estímulo de la ejecución personal, como para mostrar las posibilidades didácticas de este recurso.

En este sentido conviene señalar que en uno de los ordenadores de la UAM (VAX 11/780) se dispone de un sistema de cuentas utilizables con fines docentes bajo el control del respectivo profesor. El planteamiento adoptado consistió en la preparación de dos tipos de "logicales".

a) Un procedimiento de comandos del sistema operativo encargado de ejecutar las acciones a él demandables por los alumnos; entre ellas caben citar la impresión, borrado o visualización de los resultados obtenidos, ejecución múltiple o no del programa, control de errores, etc. Para tal fin se elaboró un procedimiento tipo menú, de tal modo que el alumno solamente debia seleccionar las operaciones deseadas.

b) El programa propiamente dicho para la realización de la proyección partía de una versión escrita en FORTRAN IV por Cole (1974), luego ampliada por Cebrián y Bosque (1982) y finalmente retocada por nosotros para convertirla en interactiva en FORTRAN 77. De esta forma se trataba de facilitar al usuario no experto la resolución del problema de cálculo, puesto que el programa a lo largo de su ejecución va demandando la información necesaria, sean datos, sean determinadas opciones.

\section{DESARROLLO DE LA APLICACIÓN Y EVALUACIÓN}

La realización práctica de la simulación implicaba preparar previamente los datos requeridos (valores absolutos y tasas del momento de partida) y establecer las hipótesis sobre la evolución, tanto del movimiento natural, como del migratorio (en caso de que se hubiese considerado). La ejecución del programa por los alumnos se desarrolló libremente durante un período de un mes aproximadamente en las salas de terminales del Centro de Cálculo, sin que se planteasen dificultades reseñables. 
La opinión global mayoritaria, mostrada tanto verbalmente, como en los informes escritos finales, coincidía en valorar de forma muy positiva el método didáctico del trabajo de curso, aunque constatando asimismo la laboriosidad exigida por la localización y uso de las fuentes estadísticas. En lo que concierne al empleo de la informática, que es el punto que aquí nos ocupa, merece la pena recoger algunos párrafos contenidos en los informes finales de los alumnos:

«El trabajo nos ha servido para llevar a cabo los pasos del método científico... el realizar un trabajo de este tipo favorece mucho más el aprendizaje..."

“... lo más interesante y de mayor valor ha sido nuestro contacto con uno de los métodos pedagógicos aplicados a la Geografía».

"Esta proyección ha sido realizada con el objeto de obtener un conocimiento más profundo de las posibles aplicaciones de la Informática en el campo de la Geografía Humana. Creemos que este objetivo ha sido ampliamente alcanzado y, además, se nos ha introducido en el mundo de los ordenadores, lo que nos resulta de gran interés personal..."

“Hemos utilizado técnicas de trabajo aprendidas de un modo teórico en las clases regulares, que nos han obligado a manejar fuentes y a realizar cálculos (incluso con ordenador), todo to cual nos ha abierto las perspectivas de una forma más moderna de entender la Geografía».

Actitudes desfavorables prácticamente no se han expresado; únicamente cabe citar alguna que hace alusión a las limitaciones del modelo matemático de la proyección (muy elemental ciertamente), por cuanto no permitía una total flexibilidad a la hora de introducir ciertos cambios en los ciclos de cálculo.

Desde la óptica del enseñante los defectos más relevantes advertidos atañen a un corto, pero decisivo número de aspectos: En primer lugar se constató una escasa justificación de las hipótesis de proyección, que traducía tanto una exigua reflexión preparatoria a la proyección, como una gran inseguridad o temor a expresar de forma objetiva y precisa las bases o parámetros del pronóstico. Ante ese síntoma cabe recordar que la vocación utilitaria o aplicada de la Geografía, hecho tantas veces proclamado, como de problemática realización en nuestro país, pasa por una formación de los estudiantes en el "arte" de diseñar escenarios futuros sobre la base del conocimiento actual y pasado. De ahí la necesidad de insistir y profundizar en ejercicios como el expuesto, ya que constituyen la via para conjugar conocimientos teóricos y aplicacio- 
nes positivas. La destreza de saber establecer hipótesis, conceptual y operativamente, sobre la evolución futura de fenómenos, es la condición indispensable para que el geógrafo pueda participar en la elaboración de estrategias de planificación.

En segundo lugar, y esta es la deficiencia más grave, se detectó un análisis parco $o$ inexistente de los resultados o escenarios proyectados. Ello traduce uno de los riesgos comúnmente citados en la literatura: el empleo del ordenador puramente por la novedad que supone y sin aprovechar el aumento en la reflexión y análisis geográfico que permite.

La corrección de estos defectos se ha intentado, en el curso de una repetición de esta experiencia, mediante la combinación de varias acciones: por una parte se ha planteado una exigencia mas firme de la labor analítica personal; por otra se han propuesto algunas hipótesis de proyección comunes a todos los alumnos, de cara a ilustrar el proceso de especificación operativa de las previsiones de crecimiento; tras ello los alumnos, de forma personal, deben establecer, al menos una tercera hipótesis de evolución, justificándola sobre la base de su conocimiento geográfico del área; finalmente se han previsto sesiones de exposición y discusión sobre las cuestiones más problemáticas (determinación cualitativa y cuantitativa del ritmo evolutivo) y sobre los hallazgos.

Amén de ello emergieron algunas pequeñas dificultades en los datos por la escala de análisis adoptada (el municipio); a ese nivel por ejemplo se carece de información publicada sobre movimientos migratorios, hecho que motivó su exclusión generalizada en los trabajos realizados; así mismo el breve periodo para el que se dispone de datos sobre movimientos naturales (aparte de los antecedentes, desde 1975) y la irregularidad interanual de esas cifras en municipios muy pequeños dificultaron el establecimiento de una situación de partida sólida para la proyección, hecho éste que se ha tratado de paliar mediante el uso de tasas medias quinquenales. El manejo de tasas de crecimiento negativas supuso finalmente algunos inconvenientes, puesto que el programa en su diseño actual se adapta con relativa dificultad a los cambios de signo en los índices utilizados para realizar los cálculos ${ }^{1}$.

Como valoración final es pertinente señalar que a lo largo de esta breve experiencia se han podido constatar un cierto número de efectos

1 Con posterioridad a esta experiencia, un equipo de geógrafos e informáticos, entre los que se halla el autor, ha elaborado un programa para el CIDE (MEC) y FUNDESCO sobre análisis y proyección de la población (cf. Bosque Sandra, J. et al., 1987). 
Lecciones de una experiencia de enseñanza de la Geografía...

positivos en la enseñanza de la Geografía derivados de varios factores: realización de tareas variadas, uso directo de fuentes de datos, aplicación de técnicas de estudio geográfico (elaboración de mapas y gráficos, cálculo de tasas y proceso de datos), contacto directo con un área concreta (que con cierta frecuencia se tradujo en visitas y encuestas informales in situ por los propios alumnos, pese a no haberse exigido), etc., tales efectos se plasmaron en un interés sostenido y en un sentimiento de satisfacción palpables. Los defectos apuntados creemos que no empañan esta conclusión; su solución parece asequible. $Y$ estimamos que profundizar en esta vía puede suponer, para el estudioso de la Geografía, por un lado un aumento en la comprensión, y sobre todo, en la capacidad de análisis de la dinámica geodemográfica, y por otro una capacitación para enfrentarse sin complejos e inseguridades con los procedimientos habituales de la planificación territorial.

\section{AGRADECIMIENTOS}

A los profesores Cebrián de Miguel y Bosque Sendrá por habernos facilitado el programa y al personal del Centro de Cálculo de la UAM por el apoyo ofrecido, tanto en asesoramiento, como en el uso de sus instalaciones. 


\section{BIBLIOGRAFÍA}

Beaumont, J. R. y Williams, S. W., 1983: Project Work in the Geography Curriculum. Londres, Croom Helm.

Bosque SendRA, J., et. al., 1987: DEMOS. Un programa de ordenador para el estudio del crecimiento de la población. Madrid, CIDE-FUNDESCO (policopiado).

Cebrián, J. A. y Bosque SendrA, J., 1982: “Un modelo multirregional para la proyección de la población de las provincias españolas". Anales de Geografia de la Universidad Complutense, 2, p. 99-125.

COLE J. P., 1974: «Proyecciones de la población de México», Bol. del Inst. de Geo., 5, p. 187-200.

FernÁndez GonzÁlez, M., 1983: Enseñanza asistida por odenador. Madrid, Anaya.

Kent, A., 1983, ed.: Geography Teaching and the Micro. Harlow, Longman, $72 \mathrm{p}$.

MidgleY, H., y WALKER, D., 1985: Microcomputers in Geography Teaching. London, Hutchinson, $197 \mathrm{p}$.

Moreno, A., et. al, 1983: "Informática y Geografía», en La Geogra-Teórica y Cuantitativa. Concepto y Métodos. Universidad de Oviedo (en prensa).

MORENO, A., 1988: “El ordenador en la enseñanza de la Geografia», en Aplicaciones de la Informática en la Geografía y las Ciencias Sociales, Madrid, Síntesis, p. 271-299.

Pentiraro, E., 1983: El ordenador en el aula. Madrid, Anaya-Multimedia.

ReEVE, D. E., 1985: "Computing in the geography degree: limitations and objectives", Jou. of Geo. in Hig. Educ., v. 9, 1, p. 37-44. 
Shepherd, I. D. H., Cooper, Z. A. y Walker, D. R. F., 1980: Computer Assisted Learning in Geography. Current Trends and Future Prospects. Londres, Council for Educational Technology.

SHEPHERD, I. D. H.: “Teaching geography with the computer: possibilities and problems", Jou of Geo. in Hig, Educa., v. 9, 1, p. 3-23.

Shavelson, R. J., et. al., 1984: Succesful Teachers' Patterns of Microcomputer-Based Mathematics and Science Instruction, Santa Mónica, The Rand Corporation, $241 \mathrm{p}$.

SILK, J. y BowLBY, S., 1981: "The use of project work in undergraduate geography teaching", Jou. of Geo. in Hig. Educ., v. 5, 2, p. 155-162.

VIDAL, M. J., Bosque, J. y MoReno, A., 1981: «Procedimientos informáticos para el análisis de datos geográficos", Anales de Geografía de la Univ. Complutense, 1, p. 333-344. 